\title{
Aktuelle systematische Reviews zu Tanz-, Bewegungstherapie mit älteren Menschen und Demenzkranken und
} ein RCT

Iris Bräuninger

D ieser Beitrag stellt zwei systematische Reviews zur Wirkung der Tanz-, Bewegungstherapie bei älteren Menschen (Jiménez et al. 2019) und älteren DemenzpatientInnen (Lyons et al. 2018) vor und präsentiert die Ergebnisse eines RCTs zur Wirkung der Tanztherapie auf die psychophysiologischen Effekte bei älteren Menschen mit milder Demenz.

\section{Systematischer Review zur Wirkung der Tanz-, Bewegungstherapie bei älteren Menschen mit}

\section{psychiatrischer Diagnose}

Jiménez, Bräuninger und Meekums (2019) führten einen systematischen Review zur Tanz-, Bewegungstherapie bei Menschen über 60 Jahren mit psychiatrischen Diagnosen durch. Die Suche wurde in den Datenbanken CINAHL, MEDLINE, PsycINFO und PSYNDEX sowie bei Google Scholar durchgeführt, zusätzlich wurden Literaturlisten von relevanten Veröffentlichungen geprüft. Eingeschlossen wurden verschiedene Arten von Evidenzstudien, deren methodische Qualität anhand des PRISMA-Flussdiagramms festgelegt und anhand der JBI Critical Appraisal Tools entsprechend der Studienart beurteilt wurden (Joanna Briggs Institute 2019). Der Review schloss quantitative und qualitative Stu- dien sowie systematische Übersichtsarbeiten zur Tanz-, Bewegungstherapie ein, welche patientenrelevante Parameter und Daten dokumentierten, aber keine Vergleichsinterventionen anbieten mussten. Ausgeschlossen wurden ExpertInnenmeinungen, nicht-systematische Literaturüberblicke und graue Literatur. Anhand der Kriterien wurden von ursprünglich 177 Studien, deren Abstract gecheckt wurden, 16 Studien im Volltext von den zwei Gutachterinnen eingeschlossen: neun Primärstudien (eine randomisierte kontrollierte Studie, drei quasi-experimentelle Studien, fünf qualitative Studien) und sieben Sekundärstudien (systematische Reviews). In 15 Studien wurde Demenz und in einer Depression thematisiert. Anhand der Checklisten wurde die methodische Qualität in vier der sieben systematischen Reviews als hoch, in zwei als mittelwertig und in einer als niedrig beurteilt. Die methodische Qualität der Primärstudien wurde in zwei von vier Studien als mittelmäßig und in zwei Studien mit niedrig bewertet. Für die qualitativen Studien wurde die methodologische Qualität in zwei von fünf Studien mit hoch, für den Rest als niedrig evaluiert. Die methodische Qualität der Primärstudien wurde im Vergleich zu den Sekundärstudien als geringer bewertet. Eine Einschränkung war, dass die meisten Primärstudien in den Sekundärstudien einbezogen waren. 


\section{Systematischer Review zum tanz-,} bewegungstherapeutischen Effekt auf Gesundheit und Wohlbefinden

\section{bei älteren Menschen mit Demenz}

Der systematische Review von Lyons und KollegInnen (2019) schloss neben quantitativen und qualitativen auch künstlerische Studien ein, welche sich auf die gesundheitliche Verbesserung von Menschen mit Demenz ab 65 Jahren durch Tanz-, Bewegungstherapie konzentrierten. Ziel der Studie war, die theoretischen Rahmen und therapeutischen Komponenten zu identifizieren, welche für die Arbeit mit DemenzpatientInnen in der Tanz-, Bewegungstherapie bedeutungsvoll sein könnten. Die Literatursuche wurde in den Datenbanken AMED, CINAHL, EMBASE, MEDLINE und PsycINFO durchgeführt. Zwei Gutachterinnen überprüften die Studien anhand von Einschluss- und Ausschlusskriterien, welche sich an den Kategorien Population, Intervention und Studiendesign orientierten. Die Auswahl und der Ablauf wurden anhand des PRISMA-Flussdiagramms definiert, die Qualität der eingeschlossenen quantitativen Studien wurde anhand des „Risk of Bias Tool“ (Higgins/Green 2011) bewertet. Die Qualität der qualitativen Studien wurde mit einem ABCD-Bewertungssystem beurteilt. Anhand der Kriterien wurden von ursprünglich 521 Studien, deren Abstracts gecheckt wurden, fünf hauptsächlich qualitative Beobachtungsstudien unterschiedlicher methodischer Qualität berücksichtigt. Die theoretischen Rahmen der eingeschlossenen Studien basierten vor allem auf dem personenzentrierten Ansatz sowie auf psychodynamischen Elementen. Als studienübergreifende therapeutische Komponenten konnten Spontaneität und Improvisation, dramatische Szenarien, rhythmische Synchronisation und Symbolik identifiziert werden. Die Studien betonten für die Arbeit mit DemenzpatientInnen die Hervorhebung wichtiger Momente und die Verbindungen von Gedanken, Gefühlen und körperlichen Empfindungen.
Lediglich eine der fünf Studien wurde mit mittelmäßigem Evidenzniveau eingestuft, alle anderen als niedrig.

\section{RCT zur Wirkung auf die psycho-}

\section{physiologischen Effekte bei älteren} Menschen mit milder Demenz

Der RCT von Ho und KollegInnen (2019) untersuchte die psychophysiologischen Wirkungen von Tanz-, Bewegungstherapie und körperlicher Übung auf ältere Erwachsene mit Demenz. Für die Studie wurden 204 PatientInnen (Durchschnittsalter 79.0 Jahre, $S D=8.0$, davon $81.9 \%$ Frauen) einer der drei Gruppen (Tanz-, Bewegungstherapiegruppe, Übungsgruppe oder Wartekontrollgruppe) zufällig zugeordnet. Zu Studienbeginn ( $\mathrm{t} 1$ ) und zu drei Follow-up-Messungen (t2: Post-Test, t3: 6 Monate nach t1, t4: 12 Monate nach t1) füllten alle Teilnehmerlnnen standardisierte Selbstbewertungsfragebögen zu psychosozialem Wohlbefinden, täglicher Funktionsfähigkeit und neurokognitiver Beurteilungen über einen Zeitraum von einem Jahr aus und ließen Speichel-Cortisol-Messungen durchführen. Die beiden Interventionsgruppen erhielten zweimal wöchentlich über 12 Wochen entweder Tanz-, Bewegungstherapie (einfache Gruppentänze, Bewegungsspiele, Tanzimprovisation und Bewegungsinteraktionen zwischen TeilnehmerInnen) oder ein einfaches Übungsprogramm (Warm-Up, Stretching, Übungen, Cooldown). Die Ergebnisse zeigten keine Unterschiede zwischen den Gruppen zu t1, jedoch eine signifikante Reduktion von Depression, Einsamkeit und negativer Stimmung $(d=0.33-$ $0.42, p<0.05)$ und eine Verbesserung der täglichen Funktion $(d=0.40, p<0.01)$ und der täglichen Cortisolsteigung $(d=0.30, p<0.01)$ im Prä-Test-Post-Test-Vergleich in der Tanztherapiegruppe. Die Effekte zu den täglichen Funktionen und zur Cortisolsteigung zeigten sich auch in der einjährigen Nachmessung. Im Vergleich zeigten sich keine signifikanten Verbes- 
serungen in der Übungsgruppe. Aufgrund des Fehlens langfristiger Tanztherapie-Effekte (mit Ausnahme täglicher und neuroendokriner Funktionen) schlugen die Autorlnnen vor, kontinuierliche Tanzbewegung im Rahmen eines strukturellen Rehabilitationsprogramms anzubieten, um ältere Erwachsene psychosozial zu unterstützen.

\section{Schlussfolgerung}

Die beiden systematischen Reviews wiesen auf methodische Defizite der Studien zu Tanz-, Bewegungstherapie bei älteren Menschen mit Demenz (Lyons et al. 2018) und der Primärstudien zur Tanz-, Bewegungstherapie bei älteren Menschen mit psychiatrischen Diagnosen (Jiménez et al. 2019) hin. Der RCT von Ho und Kolleglnnen (2019) erweiterte die Forschung zur Tanz-, Bewegungstherapie mit einer hochwertigen Primärstudie, welche kurzfristige Effekte (Verbesserung affektiver Symptomatik) und langfristige Effekte (Verbesserung täglicher und neuroendokriner Funktionen) bei älteren Menschen mit Demenz nachweisen konnte.

\section{Literatur}

Higgins, J. P.T., Green, S. (2011): Cochrane Hand book for Systematic Reviews of Interventions Version 5.1.0 (updated March 2011). The Cochrane Collaboration. In: www.cochranehandbook.org, 17.7.2019

Ho, R.T.H., Fong, T. C. T., Chanc, W. C., Kwand, J.S. K., Chiu, P. K. C., Yau, J. C.Y., Lame, L. C.W.
(2018): Psychophysiological effects of dance movement therapy and physical exercise on older adults with mild dementia: A randomized controlled trial. Journals of Gerontology: Psychological Sciences 20 (20), 1-11, https://doi. org/10.1093/geronb/gby145

Jiménez, J., Bräuninger, I., Meekums, B. (2019): Dance movement therapy with older people with a psychiatric condition: A systematic review. The Arts in Psychotherapy 63, 118-127, https://doi.org/10.1016/j.aip.2018.11.008

Joanna Briggs Institute (2019): Critical Appraisal Tools. In: joannabriggs.org/critical_appraisal_ tools, 17.7.2019

Lyons, S., Karkou, V., Roe, B., Meekums, M., Richards, M. (2018): What research evidence is there that dance movement therapy improves the health and wellbeing of older adults with dementia? A systematic review and descriptive narrative summary. The Arts in Psychotherapy 60, 32-40, https://doi.org/10.1016/j. aip.2018.03.006

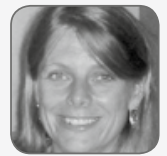

\section{Dr. Iris Bräuninger}

Senior Researcher \& Co-Leiterin Studiengang Psychomotoriktherapie (Hochschule für Heilpädagogik Zürich IVE),

Dozentin MA Tanztherapie UAB Barcelona, BTD Supervisorin / Ausbilderin / Lehrtherapeutin, KMP Notatorin, Praxis Tanztherapie Supervision Bodensee.

Dr. Iris Bräuninger dancetherapy@mac.com und iris.braeuninger@hfh.ch 